\title{
DESIGN ELEMENTS IN FLEXIBLE DIGITAL MODEL (MFD) COURSES FOR STUDENT ENGAGEMENT IN INDUSTRIAL AND SYSTEMS ENGINEERING PROGRAM
}

\author{
Resendez-Maqueda LF*, Sandoval-Correa A, Forte-Celaya MR and \\ Swain-Oropeza R \\ Tecnologico de Monterrey, Mexico
}

\begin{abstract}
Crisis caused by COVID-19 forced academics to transform Face-to-face education into digital education. As most of the courses had been never taught this way, professors needed to learn how to interact with students, and teach the academic content within a virtual platform. There was a major concern about how these online synchronous sessions had to be delivered, as well as which elements were critical for knowledge transfer and add value to the Industrial and Systems Engineering (ISE) courses. For having a proper feedback about this format, an instrument was created to evaluate the design course elements, based on theoretical approaches of teaching and learning processes in higher education, particularly on student experiences. The instrument consisted of a survey applied to ISE students from Tecnologico de Monterrey. The analysis considered the nature of the different courses involved in the study, and their categorization as Theoretical, Numerical or Mixed. After applying statistical analysis, some of those elements showed a positive evaluation by students and considering the correlations among them, recommendations for the design of Digital Flexible Model (MFD) courses are made. A positive correlation has been found between active participation of the students, voluntary participation, and the sense of involvement, as well as the ease of interacting that the technological platform provided to the students. Among other results, highlighting the usage of a technological platform is not enough to deliver MFD courses, it is necessary to consider design elements for engaging and motivating students' participation. It is notorious for the need for a "holistic" transversal approach that complements the design of the successful interaction experience of students in the MFD.
\end{abstract}

Keywords: Digital Education, MFD (Digital Flexible Model), Education in pandemic, Educational Innovation, Higher Education, Students engagement.

\section{Introduction}

The COVID-19 pandemic revolutionized the way of living and thinking of every person in all countries around the world. and knowledge transfer was not the exception. Education needed to adapt in a faster way to keep serving its purpose of delivering knowledge to students in all levels and in several countries facing different challenges, such the case of Switzerland where higher education turned online during all the semester (Kovacs, Pulfrey, \& Monnier, 2021) and Nigeria where the institutions, infrastructure, and student's preparedness for remote and e-learning was an issue (Bolu et. al, 2020) to cite a couple of them. In the case of Mexico, on March 12th, 2020, the university Tecnologico de Monterrey declared the suspension of face-to-face classes as a prevention although 
there were not cases in the institutional community; also, compromised to reactivate classes on March 23rd, 2020, in a virtual way for indeterminate time (Redacción CONECTA, 2020). This gave the faculty staff of this private university just a week to prepare, change and redesign their courses for being deliver through a virtual platform according to the Flexible Digital Model - MFD by its acronym in Spanish courses (Tecnologico de Monterrey, n.d.).

Since Internet appeared, online learning has been in continue growth and it can be found many literatures about the experiences and recommendations about this type of delivery method (Hernández Godoy, Fernández Morales, \& Pulido, 2018; Volery \& Lord, 2000; Cohen \& Jackson-Haub, 2020) which allows to be fully asynchronous; also the traditional blended courses has been implemented in the past with a combination of online sessions and face-to-face instructions in classroom (Sahara et. al, 2021). MFD courses differs to online and blended courses because it includes both synchronous and asynchronous sessions, trying to emulate what happens in face-to-face education, which due sanitary restrictions derived of the pandemic cannot be in a physical space; like blended online learning (Fadde \& Vu, 2014). MFD also includes increases flexibility, use of technology and active learning (Tecnologico de Monterrey, n.d.). The activities adaptation to this delivery mode and training in the use of the platforms were some of the challenges that most of the professors in the institution faced as they did not have the experience of teaching either remote or online courses.

When the contingency began, the authors of this paper were concerned about the impact that online education could have over the student's performance and the course's adaptation to the new remote format. According to Noteborn et.al (2012) research, emotions impact how students enjoy learning and influence their attitudes towards virtual education, also Ferrer et. al (2020) suggest that a positive attitude is related to student's motivation, intrinsic motivation helps to feel well about learning and engage them to learn in different contexts, and extrinsic motivation drive some engagement to learning activities. Considering this, it was agreed to focus on elements that could improve the student's motivation and engagement in the Industrial and Systems Engineering (ISE) disciplinary courses.

This paper discusses some elements that were identified by students in the MFD courses designed by professors and the influence of the items in the student's motivation to virtual courses. A survey with 27 items was designed and applied to the $87 \%$ of the ISE program at Tecnologico de Monterrey in Sonora, Mexico the last week of the semester. The study considers the course classification and suggest some proposals to implement in the design of MFD courses to motivate and engage students. The recommendations could help professors that will continue teaching in a remote mode to redesign their classes to a digital version.

\section{Literature Review}

Online learning, described by Rhim and Han as "access to learning experiences via the use of the Internet," is an evolution of the idea of distance learning (Rhim, H.C., \& Han, H., 2020, p. 176). Due to the COVID-19 pandemic, educational institutions were forced to speed up and adapt their various instructional contents to deliver them to their students.

Beyond sharing information through a different channel, it is keeping students' interest in the subjects, their willingness to engage and share their ideas, and providing a virtual environment where knowledge can be created, the challenges that educators have had to face because of the emergency. 
This is where the design of online sessions, as well as the design of courses and teaching methods, become extremely important. In addition, Rhim and Han (2020) suggest that online learning can be divided into three foundational principles for evaluating its effectiveness: "Transactional Distance", "Presence", and "Independent Learners". Moore (1993) describes transactional distance as a fundamental phenomenon that is linked to social, psychological, and relational differences between teachers and students. When dialogues, or constructive interactions between teachers and learners for the development of information, are increased, this gap can be bridged.

The instructor should be able to monitor and handle the amount and essence of each experience by introducing and reinforcing these elements during the course design. In an online learning environment, faculty should provide opportunities for student reflection, consider various mediums for content delivery, and emphasize that learning requires elements of high-impact teaching, which enhances faculty-student engagement, affects overall course interactions, and outcomes, according to Montelongo (Montelongo, 2019).

According to Rhim and Han (2020), the foundational concept "Presence", is further subdivided into cognitive, social, and teaching presence. The first refers to learners' sense creation by constant exchange of thoughts; the second to learners' personal feelings and emotions; and the third to educational experience design and facilitation (Anderson, 2008). Finally, the idea of independent learners refers to a student's ability to develop or change learning methods to produce the best results. But it is Henry (2020) who claims that universities will be able to improve student satisfaction and retention by knowing what online students expect. Six main themes were used to characterize desires, according to him: motivation, ability, circumstances, interaction, curriculum, and environment. Particularly, motivation in participants, described their intrinsic desire to engage with their learning.

Literature analysis leads to approaches that present design ideas based on students' responses for teaching and learning in higher education, with an emphasis on the student experience (Wood \& Shirazi, 2020) in our quest for the most suitable designs of Digital and Flexible Courses.

Wood's research improves concepts that should be considered for a desired audience response and is organized into a three-layer model: 1) the students; 2) the learning environment; and 3) additional factors; 18 different elements are detailed mapped in each of these layers as shown in Figure 1. Even if those considerations involve the use of technology during classes, they do not represent the effect of those considerations in a Digital and Flexible Course. More specifically, a comprehensive category of characteristics identified as critical success factors in online education include factor analysis of technology, factor analysis of instructor characteristics, and factor analysis of student characteristics (Volery \& Lord, 2000).

Another approach focuses on online education and is more focused on student engagement, considering factors like social engagement, cognitive engagement, behavioral engagement, collaborative engagement, and emotional engagement (Cohen \& Jackson-Haub, 2020). Other research on online environments and interactions suggests that some course factors, such as content style, structure, and interaction tools, should be considered, as well as some instructor factors, such as instructional design and assignments (Lear et al, 2010). 
A survey was applied to students to find the elements that increased their involvement during their participation in Flexible Digital Model (MFD) Courses, based on the previously listed works and considering those approaches for every question planned.

\section{Research Objectives}

The general purpose of this study is to identify and provide information about the best practices and design characteristics that should be included in the Industrial Engineering courses within a synchronous face-to-face online session. The specific objectives are mainly two:

1. Identify if there are some significant differences in the design elements that motivate and engage Industrial and Systems Engineering students regarding the course structure and content classification (numerical, theoretical, and mixed).

2. Make proposals about how to implement the design elements considered as motivating by the students in the MFD model proposed by Tecnológico de Monterrey.

\section{Methodology}

Considering that courses were adapted from Face-to-face to digital format, the research group inquiry about the elements that students preferred and evaluated the better during synchronous sessions, so it can derive in enhancing their engagement and participation in class. As engagement it is an important aspect to academic performance and outcomes (Christenson, Reschly \& Wylie, 2012) the authors looked for the relationships between those present and well evaluated aspects in online synchronous sessions to find some key elements that could be useful for professors when redesigning their courses.

The first step in the process was to identify previous studies about good practices regarding online education such as considering interaction tools and teachers' presence (You \& Beal, 2017), search for elements that could represent key indicators to evaluate engagement in students (Cohen \& JacksonHaub, 2020;) and define how to make a diagnosis about their current motivation levels. Also, it was necessary to identify the characteristics of the official model used by Tecnologico de Monterrey to deliver the content of its online courses and the design of the synchronic academic sessions. This institutional model is known as Digital Flexible Model or MFD by its acronym in Spanish includes flexibility, easy to continue classes by webconference, active learning, use of technology, continuous feedback among other elements (Tecnologico de Monterrey, n.d)

After defining these characteristics and completing the academical search for good practices within online education, the research and instrument for obtaining the desired information was designed.

As it was the first time that students took MFD courses and it was not a control group because all the classes turned to this model as a response of the pandemic, a posttest-only non-experimental design research was conducted. With the purpose of identify the rating of the different items that could be present in the synchronous session, describe them with descriptive statistics and find some correlations between elements and level of participation and motivation; it was decided to use a quantitative analysis and considering it is social research about perceptions and opinions of students a survey method was selected for data collection (Trochim, Donnelly \& Arora, 2016) 
The survey was created considering the theoretical background regarding engagement. The questions were chosen and designed by the research team to evaluate digital courses and get student's answers/evaluations considering the elements and three layers: students, learning environment, and some additional factors, each with their corresponding subclassification of elements as proposed by Wood and the corresponding classification for engagement proposed by Cohen; as shown in Figure 1 (Wood, 2020).

\begin{tabular}{|c|c|c|c|c|c|c|c|c|c|}
\hline \multirow{3}{*}{ Q1 } & \multicolumn{4}{|c|}{ Wood's Layer } & \multicolumn{5}{|c|}{ Cohen's Classification } \\
\hline & Element & $\begin{array}{l}\text { The } \\
\text { Student }\end{array}$ & $\begin{array}{c}\text { Learning } \\
\text { Environment }\end{array}$ & $\begin{array}{c}\text { Additional } \\
\text { Factors }\end{array}$ & $\begin{array}{c}\text { Emotional } \\
\text { Engagement }\end{array}$ & $\begin{array}{l}\text { Collaborative } \\
\text { Engagement }\end{array}$ & $\begin{array}{c}\text { Cognitive } \\
\text { Engagement }\end{array}$ & $\begin{array}{l}\text { Behavioral } \\
\text { Engagement }\end{array}$ & $\begin{array}{c}\text { Social } \\
\text { Engagement }\end{array}$ \\
\hline & Anonymity & & & $\bullet$ & 0 & & & & \\
\hline Q2 & Anonymity & & & - & 0 & & & & \\
\hline Q3 & Anonymity & & & - & 0 & & & & \\
\hline Q4 & Participation & $\bullet$ & & & & 0 & & & \\
\hline Q5 & Participation & $\bullet$ & & & & 0 & & & \\
\hline Q6 & Questons Design & & & $\bullet$ & & & 0 & & \\
\hline Q7 & Pace of Learning & & & - & & & & 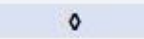 & \\
\hline Q8 & Integration & & & - & & 0 & & & \\
\hline Q9 & Participation & - & & & & 0 & & & \\
\hline Q10 & Participation & - & & & & 0 & & & \\
\hline Q11 & Organization of the Cohort & & & - & & & & & 0 \\
\hline Q12 & Collaboration & - & & & & 0 & & & \\
\hline Q13 & Active Learning Environment & & - & & & & & & 0 \\
\hline Q14 & Active Learning Environment & & - & & & & & & 0 \\
\hline Q15 & Discussion & & - & & & & & & 0 \\
\hline Q16 & Competition & & • & & & & & & 0 \\
\hline Q17 & Sense of Involvement & $\bullet$ & & & & & & & 0 \\
\hline Q18 & Confidence & - & & & 0 & & & & \\
\hline Q19 & Active Learning Environment & & - & & & & & 0 & \\
\hline Q20 & Self-directed Learning & & $\bullet$ & & 0 & & & & \\
\hline Q21 & Immediacy of Feedback & & $\bullet$ & & & & 0 & & \\
\hline Q22 & Self reflection & - & & & 0 & & & & \\
\hline Q23 & Self assessment & - & & & 0 & & & & \\
\hline Q24 & Self-directed Learning & & - & & 0 & & & & \\
\hline Q25 & Metacognition & - & & & & & 0 & & \\
\hline Q26 & Metacognition & - & & & & & 0 & & \\
\hline Q27 & Teacher's response to questions & & & - & & & 0 & & \\
\hline
\end{tabular}

Figure 1: Relationships between questions and Woods' elements and layers, and Cohen's Classification.

It consisted in a survey that looked for relevant information about the design, performance, and most valued characteristics in an Industrial and Systems Engineering course.

The online survey was applied to a non-probabilistic sample to students enrolled in 10 different courses of Industrial and Systems Engineering (ISE). The courses were selected by convenience for the research considering their nature to represent accurately the different nature of the complete set of subjects, also it was considered the number of students enroll in the courses to have a good percentage of the student's population answering the survey. The survey evaluated in a quantitative way the 27 items using a Likert scale in a range from 1 to 5 , being 5 the highest score for evaluation.

\section{Description of course classification considering their nature.}

To compare the impact of the different characteristics evaluated, it was also considered a description of course classification considering their nature. It was decided to categorize them in three main groups: numerical, theoretical, and mixed. 
- Theoretical: courses that have more theory and have a philosophical approach, usually, can be taught as a lecture. These courses are based on conceptual models, abstract thinking, or non-practical methods.

- Numerical: courses that need to do calculations, usually students are asked to solve numerical problems. Subjects whose focus is based on mathematical approaches or problem-based learning.

- Mixed: courses in which the content has some balance between theory and practice (simulations, some calculations, etc.)

\section{Data collection}

The survey was applied to students enrolled in different Industrial and Systems Engineering courses at the end of the "February-June 2020" semester, this means, during the first period of the pandemic when professors and students were adapting themselves to digital courses. The head of the ISE department asked professors to apply the survey in their groups of the select courses. In turn, professors asked their students during the class to answer the online survey and share with them the link, at the same time the program director reinforce the request via e-mail to students enroll in the courses selected. Students were asked to answer and evaluated considering only their experience in the specific course select in the list of subjects that appeared in the questionnaire. Students were able to evaluate more than one course by sending different forms. Institutional e-mails were collected automatically when the form was submitted to identify is there were duplicity of answers for one course/subject. 89 students answered who represented the $87 \%$ of the ISE population in the campus.

\section{Analysis}

Once the information was gathered, a statistical analysis was carried out to identify the descriptive statistics and if there were some correlations between the questions, which could lead to the creation of some hypothesis about the design elements that every MFD course that should be included to motivate and engage students. At first, a general analysis was made, without considering the course classification. The correlation analysis was considered to identify which elements could be related and how they could influence each other and conclude some design course tips to potentiate the participation and engagement of students.

A Two Sample T-Test was conducted to identify if there was any difference between the mean evaluation of each question considering the course classification. The sample for numerical courses was 73 opinions, for theoretical was 39 and for mixed 31. The method for each evaluation was considering $\mu \mathrm{x}$ : population mean of Px considering the course type. First it was compared numerical $\left(\mu_{1}\right)$ and theoretical $\left(\mu_{2}\right)$, then mixed $\left(\mu_{1}\right)$ and numerical $\left(\mu_{2}\right)$; at last, the evaluation for mixed $\left(\mu_{1}\right)$ and theoretical $\left(\mu_{2}\right)$ was made. For all cases, a $95 \%$ of confidence was considered and the following hypothesis:

\section{Null hypothesis $\quad H_{0}: \mu_{1}-\mu_{2}=0$; Alternative hypothesis $\quad H_{1}: \mu_{1}-\mu_{2} \neq 0$}

The result was useful to know if some elements were more important, valuable, or appreciated in one type of course or another. 


\section{Results}

Even when every question was designed based on the Wood and Cohen approaches for a successful digital course, not in all questions the students' answers confirm a level of success by itself. It is possible to identify a set of questions whose assigned evaluations, according to the students' perception, confirm a level of participation and motivation that could imply engagement: Q1, Q2, Q3, Q4, Q5, Q9, Q10, Q16, Q17, Q18. On the other hand, it is possible to identify a set of questions whose assigned evaluation confirm only the element's presence in the course: Q6, Q7, Q8, Q11, Q12, Q13, Q14, Q15, Q19, Q20, Q21, Q22, Q23, Q24.

A descriptive analysis was made for identifying the rating obtained from each question regardless of the course classification as show in Figure 2.

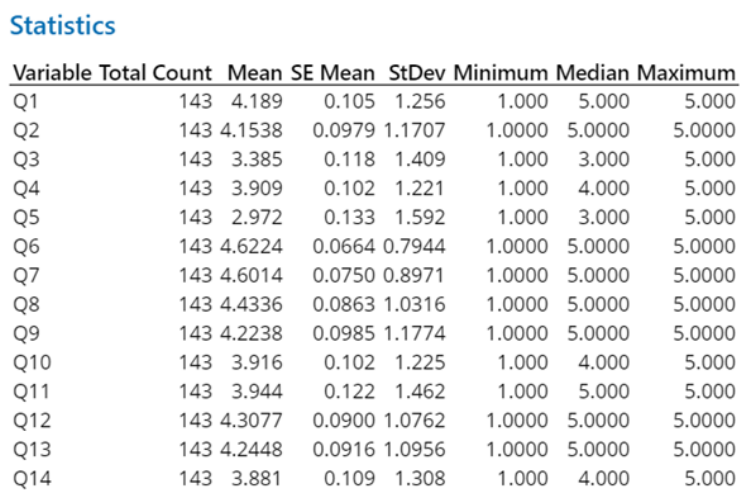

\begin{tabular}{lrrrrrrr} 
Statistics \\
\multicolumn{7}{l}{ Variable Total } \\
\hline & 143 & 4.007 & 0.102 & 1.219 & 1.000 & 4.000 & 5.000 \\
\hline Q15 & 143 & 3.580 & 0.117 & 1.401 & 1.000 & 4.000 & 5.000 \\
Q16 & 143 & 3.958 & 0.108 & 1.294 & 1.000 & 4.000 & 5.000 \\
Q17 & 143 & 4.1259 & 0.0997 & 1.1919 & 1.0000 & 5.0000 & 5.0000 \\
Q18 & 143 & 4.5734 & 0.0691 & 0.8264 & 1.0000 & 5.0000 & 5.0000 \\
Q19 & 143 & 3.811 & 0.105 & 1.250 & 1.000 & 4.000 & 5.000 \\
Q20 & 143 & 4.3776 & 0.0786 & 0.9405 & 1.0000 & 5.0000 & 5.0000 \\
Q21 & 143 & 4.2867 & 0.0950 & 1.1360 & 1.0000 & 5.0000 & 5.0000 \\
Q22 & 143 & 4.1958 & 0.0938 & 1.1212 & 1.0000 & 5.0000 & 5.0000 \\
Q23 & 143 & 4.042 & 0.105 & 1.250 & 1.000 & 5.000 & 5.000 \\
Q24 & 143 & 4.091 & 0.101 & 1.210 & 1.000 & 5.000 & 5.000 \\
Q25 & 143 & 3.706 & 0.111 & 1.331 & 1.000 & 4.000 & 5.000 \\
Q26 & 143 & 3.916 & 0.129 & 1.541 & 1.000 & 5.000 & 5.000 \\
Q27 & & & & & & &
\end{tabular}

Figure 2: Descriptive Statistics for each question regardless of the course classification

For the second set of questions that only identify the presence of the element it can be noticed that Q6 - In your class, were there moments / activities that allowed you to interact with your teacher, ask him questions and have answers? - has one of the highest evaluations with a mean of 4.6224. Which confirmed that the teacher included this aspect during his/her course. A further analysis might reveal if, for example, Q6 is one of the reasons that explains Q2 - I am motivated to participate and interact in the class session, once a correlation of both might be identified.

Also, it is possible to realize that mean evaluations vary from medium to high, despites Q5 that can be considered with a low evaluation (2.972), which in this case indicates that students are not ashamed to share their video or not like to do it.

In Figure 3, it is shown the identification of strongest correlations ( 0.6 and above) between questions regardless of course classification, particularly a total of 27. These correlations were identified with the cases of Q1 related with Q2, Q12, Q17 and Q23; for Q2 a relationship with Q1, Q4, Q6, Q17, Q18 and Q21. Other strong correlations were found for Q3 with Q4, Q17, Q13, Q17 and Q18; the Q4 is strongly related with Q2, Q3 Q13, Q17 and Q18; for Q6 the marked correlation was with Q7 and Q8; also, it was noticed Q9 and Q10. Other relationships are Q11 and Q27, Q13 with Q17 and Q18; also, Q14 with Q15, Q17 with Q1, Q2, Q3, Q4, Q13, Q18. The correlations for Q19 are with Q22 and Q23. At last, the correlations above 0.6 for Q21 where with Q22 and Q23, also Q22 with Q23. 


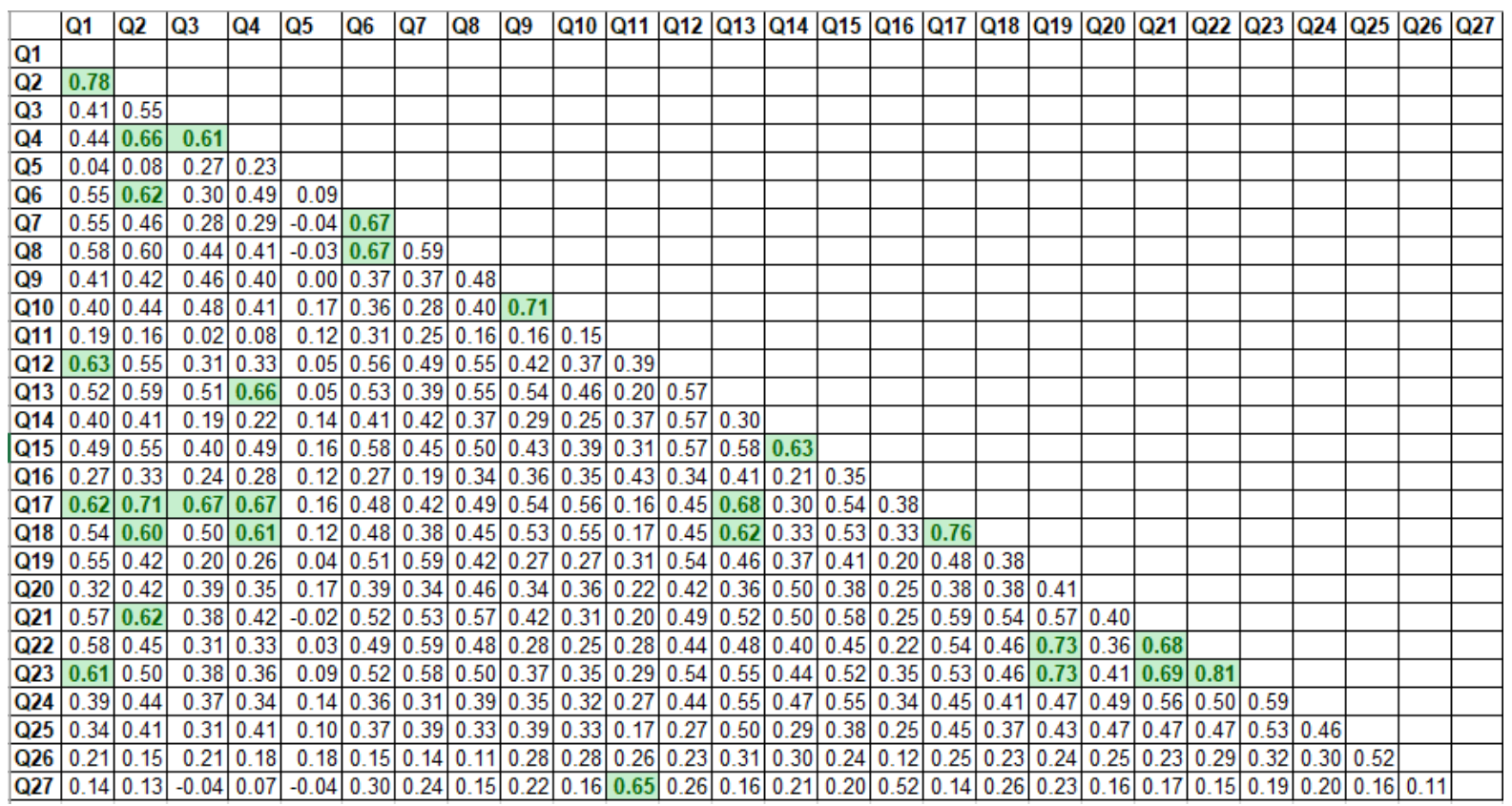

Figure 3: Identification of strongest correlations between questions regardless course classification

The questions related to Q2 provide us with elements that clarify why the student points out "I feel motivated to participate and interact in the class session". It is noteworthy that what is associated with Q1, Q4, Q6, Q17, Q18 and Q21 which corresponds to different factors and layers according to the Wood and Cohen models (Cohen, 2019, Wood, 2020) which highlights the need for a cross-sectional approach or "Holistic", that complements the design of the successful interaction experience of students in the MFD. Considering the three layers (the student, learning environment and, additional factors), together with the elements of participation, question design, immediacy of feedback, sense of involvement, confidence and anonymity. Which can impact the collaborative, cognitive, social, and emotional engagement.

In the case of Q4, "How often did the students participate voluntarily during the class", it can be observed its relation to the questions Q2, Q3 Q13, Q17, and Q18. Again, the three layers are involved, and the elements anonymity, active learning environment, sense of involvement, and confidence appear. But in terms of classification only emotional engagement and social engagement are present.

The questions Q1, Q2, Q3, Q4, Q13, Q18, related to Q17 provide us with information that allows the understanding of why the student points out "The technological platform allowed you to feel truly involved and to be able to participate and interact appropriately with your classmates and teacher in the synchronous sessions". These correlations indicate that the factors that can influence the opinions correspond to different factors and layers according to cited theorists as happened with Q2, which also highlights the need for a cross-sectional approach to a successful design of students' experience in the MFD. The elements that can be identified correspond also to the three layers: the student, the learning environment, and additional factors, considering the active learning environment, anonymity, participation, and confidence; all these involving the emotional, social and collaborative engagement.

In a set of correlation that includes a more different group of characteristics the question Q23 - In your class, were there moments / activities that led you to self-assess and feedback your own learning, 
and reflect on it? It can be observed in its relation to the questions Q1, Q19, Q21 and Q22. The elements that can be identified correspond also to the layers: emotional, behavioral, and cognitive engagement that focuses on students and an appropriate learning environment.

When comparing the descriptive statistics for each type of course in each question that resulted the more correlated to identify if this could differ depending on the classification; it can be found that in all cases mixed courses have a greater evaluation, and in most of the cases are followed by numerical and then theoretical as shown in Figure 4.

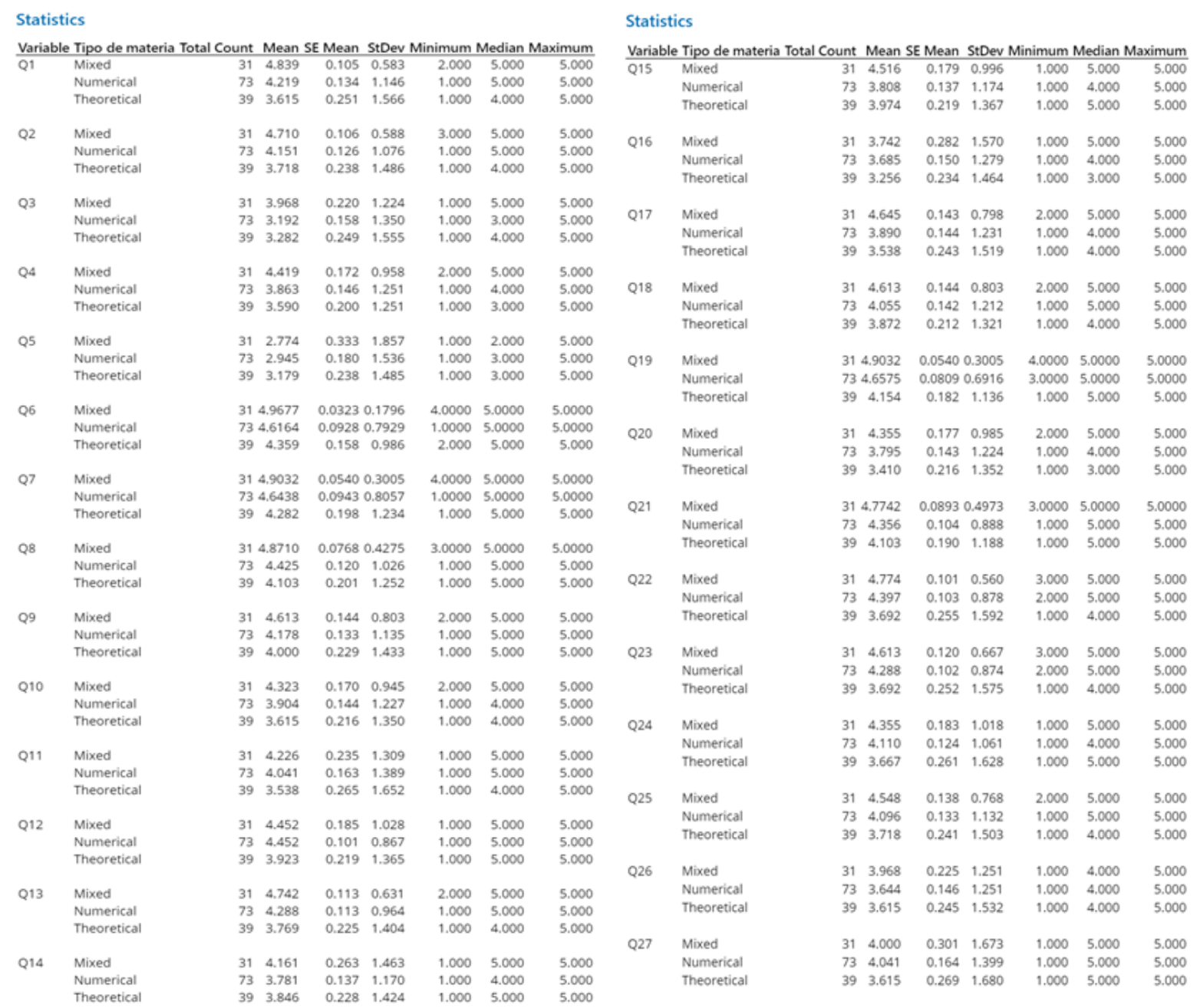

Figure 4: Descriptive Statistics for questions 1 to 27 considering course classification.

The results of the analysis made to find if these differences were significant are shown in Table 2, where the black dot represents a significant difference and the white dot indicates that no significant difference was found. Where the most significant differences were found was in the comparison of mixed and theoretical courses, followed by mixed and numerical analysis and at last with fee difference detected the analysis of numerical and theoretical courses. For Q1, there is a significant difference between the opinions considering the course type, indicating that students felt more motivated to be present in the class session in a mixed course rather than in a theoretical one. When considering the motivation to participate in class $(\mathrm{Q} 2)$ there is no difference between theoretical and numerical courses, which were evaluated with medium-high score, compared with a higher score for mixed courses that also were more consistently evaluated with a high rate. When students were asked 
if they had been participating more actively in an MFD course, it was also identified a significant difference between the mixed courses and theoretical and numerical ones, but in this case the scores for this question (Q3) were medium-high and medium-low, respectively.

Table 2: Identification of significant differences between means considering type course.

\begin{tabular}{|c|c|c|c|c|c|c|c|}
\hline Q\# & $\begin{array}{l}\text { Numerical } \\
\text { vs } \\
\text { Theoretical }\end{array}$ & $\begin{array}{l}\text { Mixed vs } \\
\text { Numerical }\end{array}$ & $\begin{array}{l}\text { Mixed vs } \\
\text { Theoretical }\end{array}$ & Q\# & $\begin{array}{l}\text { Numerical vs } \\
\text { Theoretical }\end{array}$ & $\begin{array}{l}\text { Mixed vs } \\
\text { Numerical }\end{array}$ & $\begin{array}{l}\text { Mixed vs } \\
\text { Theoretical }\end{array}$ \\
\hline Q1 & $\bullet$ & $\bullet$ & $\bullet$ & Q15 & 0 & $\bullet$ & 0 \\
\hline Q2 & 0 & $\bullet$ & $\bullet$ & Q16 & ० & 0 & 0 \\
\hline Q3 & 0 & $\bullet$ & $\bullet$ & Q17 & 0 & $\bullet$ & $\bullet$ \\
\hline Q4 & 0 & $\bullet$ & $\bullet$ & Q18 & 0 & $\bullet$ & $\bullet$ \\
\hline Q5 & 0 & 0 & 0 & Q19 & $\bullet$ & $\bullet$ & $\bullet$ \\
\hline Q6 & 0 & $\bullet$ & $\bullet$ & Q20 & 0 & $\bullet$ & $\bullet$ \\
\hline Q7 & 0 & $\bullet$ & $\bullet$ & Q21 & 0 & $\bullet$ & $\bullet$ \\
\hline Q8 & 0 & $\bullet$ & $\bullet$ & Q22 & $\bullet$ & $\bullet$ & $\bullet$ \\
\hline Q9 & 0 & 0 & $\bullet$ & Q23 & $\bullet$ & 0 & $\bullet$ \\
\hline Q10 & 0 & 0 & 0 & Q24 & 0 & 0 & • \\
\hline Q11 & 0 & 0 & 0 & Q25 & 0 & $\bullet$ & $\bullet$ \\
\hline Q12 & $\bullet$ & 0 & 0 & Q26 & 0 & $\bullet$ & 0 \\
\hline Q13 & $\bullet$ & $\bullet$ & $\bullet$ & Q27 & 0 & 0 & 0 \\
\hline Q14 & 0 & 0 & 0 & & & & \\
\hline
\end{tabular}

This can indicate that the MFD does not necessarily promote by itself and active participation of students, it is important to identify, which characteristic is responsible for it. For the question Q4 During your class, how often did you participate voluntarily? - There was no difference between opinions in numerical and theoretical courses, but there was for mixed and these to classification and evaluations tends to be medium - high; so, it can be suspected that elements in mixed courses can derive a greater willingness to participate. The question about the moments / activities that permit interaction with the professor (Q6) mixed courses were highly evaluated with almost no deviation in the opinions and a significant difference with the other two types of courses.

For Q13 that refers to the active participation of the students through solving exercises, teamwork and/or discussion, the mean evaluation differs if we compare types of courses. It was found a significant difference in the three evaluations: numerical - theoretical, where numerical got higher evaluations; in mixed - numerical and mixed - theoretical the higher evaluation was for mixed courses. This indicates that mixed courses tend to have more activities where students can participate more actively than other courses. Considering the students' feeling about being involved by the technological platform and if it allowed them to participate and interact with classmates and professors in a synchronous session (Q17), also mixed courses were highlight with better evaluations, opinions for numerical and theoretical (medium evaluations) courses did not differ but when comparing them with mixed ones (high evaluations) it did. This can be associated with the relationship with other elements not just because of the platform but with the elements included in the design of the course. Same happened when comparing opinions for Q18 which evaluates if the technological platform used for the synchronous sessions made them feel comfortable and safe to 
share their opinions or doubts, where mixed courses differed from numerical and theoretical ones. Also, for Q21 that asked about the frequency of professor feedback, the opinions for mixed courses were higher than in numerical and theoretical courses, although the three obtained high evaluations, the students for mixed courses were more consistent in their answers. This aspect could influence the opinions of other questions.

According to the research findings, when designing a MFD course some proposals can be considered to motivate and engage students, for example, include specific moments of interaction between students and teachers, like discussing open questions or debates, to reinforce the participation levels and to increase motivation. As mentioned by Bolliger and Martin (2020) in their study, the inclusion of this kind of interaction windows like discussion forums, journal entries, or reflection moments, improve the "peer engagement" between students and teachers.

Another proposal could be to include teamwork activities that require the use of technology, this will enhance the feeling of being present and facing a common objective, resulting in more engaged students. Bolliger and Martin (2020) also found in their study that the different strategies involving interactions between students and the use of synchronous web conference technologies impacted positively the "multimodal engagement", which refers to the engagement shown when different modes of content delivery are used in an online session like text, audio, video, images, etc.

One last proposal would be the use of academic technological platforms that can provide continuous feedback about exercises or activities to let students to self-asses their knowledge and compare their progress. Lu et. al (2020), mentioned that even when a student could not meet a teacher in person, they are willing to interact in an online format because they still can receive feedback in time and feel that their personal needs are better addressed.

\section{Discussion}

According to Wankel and Blessinger (2013), in blended learning, students feel more comfortable and engaged in their courses when they participate using technological tools. Also, Conrad and Donaldson (2011) related engagement with social cognition, constructivism, and an active and problem-based learning where instructors facilitate the environment. The results obtained in this research are aligned with both approaches, the correlation between the use of technology, the student participation, and the challenging activities found in some of the course's design shown a higher level of motivation and engagement among students.

Also, Richardson, York, and Lowenthal (2014) agreed that improving online interaction impacts positively the overall experience and encourage students to enroll and complete online courses. The proposals made from this research focused on reinforcing the design of MFD courses based on including different types of interaction moments between students and teachers using a technological platform.

It is important to clarify that the MFD courses appeared as a solution for ensuring continuity in education due to the pandemic COVID-19 restrictions. The results given by the students are under the context of not having real options to continue with their studies. In a regular situation, the students have a preference towards Face-to-face education due to several factors like learning environment, socializing, psychological state, and experience, among others (Kanik, 2021). 
More research and data will be collected in the future to validate if these proposals have a positive impact on the course's evaluation and to confirm if the students feel more motivated and engaged with the Industrial and Systems Engineering Program.

\section{Limitations}

The analysis and results presented are limited to a group of Industrial and Systems Engineering students in a private college located in Northwestern Mexico, different results can be found with different populations. Further studies should include a wider population, comparing the differences between regions and study programs.

Also, it must be considered that the survey was applied during the first months of the pandemic, the results can differ from others that can be obtained when gathering information in future stages of the pandemic. This can happen if we consider factors such as: professor experience with MFD courses, emotions about the pandemic and the students over exposition to digital courses. Future analysis should compare opinions over time.

The study does not include the design of the teaching methodology of each course, so results can be influenced by the course design made by each professor.

The results obtained from the comparison between types of courses cannot be interpreted as a negative evaluation for numerical and theoretical courses as compared to mixed ones because they also obtained good evaluation from medium to high. The objective of the comparison and analysis is to identify if the nature of the course influenced the opinions and notice the importance of considering the course type when designing a course, and the elements that can be included to improve students' participation and motivation. The analysis just observed if courses evaluations differed, next studies should derive in looking for correlations to include recommendations for each classification.

\section{Conclusions and Recommendations}

In Flexible Digital Model Student Motivation to participate and interact in the class session throughout the technological platform, is directly correlated with the inclusion of moments / activities that allowed a student to interact with his/her teacher, ask him questions and have answers as well as get feedback from the teacher. It also motivates students to be present and to participate voluntarily, feeling involved comfortable and safe to express her/his opinions and doubts. This design includes named elements considering Emotional, Cognitive, Social and Collaborative Engagement that focuses on students and an appropriate learning environment.

Considering if in a MFD course the technological platform allowed student to feel truly involved and to participate and interact appropriately with her/his classmates and teacher has a positive correlation to the fact that the design of the course includes solving exercises, discussing with colleagues, teamwork, etc., which motivate students to participate and interact more actively and to be present in the class session, feeling comfortable and safe to express her/his opinions and doubts.

This design includes named elements considering emotional, and social engagement that focuses on students and an appropriate learning environment. 
About the voluntary students' participation in a MFD course, also a positive correlation is found with the aspect of solving exercises, discussing with colleagues, solving team activities, etc. Deploy such that students feel motivated, involved, and comfortable and safe to express her/his opinions and doubts. This design includes named elements considering emotional, cognitive, and social engagement that focuses on students and an appropriate learning environment.

Additionally, the inclusion of activities that led students to self-assess and feedback her/his own learning and reflect on it, correlates also with including activities that made the student realize the degree of progress in her/his learning and hooked to continue in that process. All of it supported by teacher explanations, exercises, and frequent feedback to students, having a positive impact in the student's motivation to be present in class. This design includes named elements considering emotional, behavioral, and cognitive engagement that focuses on students and an appropriate learning environment.

The evaluation of students for each question is influenced by the course type. In this case, the mixed courses tend to have better evaluations than numerical and theoretical ones. This indicates that some elements were more included in the design of the mixed courses that could derive a positive reaction about motivation and participation. It can be suggested that numerical and theoretical courses incorporate elements that are found in mixed courses. Future studies will be focused on the influence of the elements that work for each type of course to increase student engagement.

The redesign of a course often is a challenging experience for professors and when done in it from a face-to-face course into a synchronous digital more elements have to be considered. The findings presented in this article can help professors to redesign their courses with some elements that potentiate voluntary participation and motivation to interact during the class and being aware of the impact of the nature of the course.

\section{References}

Anderson T. Teaching in an online learning context. In: Anderson T, ed. The Theory and Practice of Online Learning. $2^{\text {nd }}$ ed. Edmonton, Canada: AU Press; 2008:343-366.

Doris U. Bolliger, \& Florence Martin. (2020). Factors underlying the perceived importance of online student engagement strategies. Journal of Applied Research in Higher Education, 13(2), 404-419. https://0-doi-org.biblioteca-ils.tec.mx/10.1108/JARHE-02-2020-0045

Bolu, C. A., Azeta, J., Mallo, S. J., Ismaila, S. O., Dada, J. O., Aderounmu, S., Ismail, A., \& Oyetunji, E. (2020). Engineering Students' Virtual Learning Challenges during COVID-19 Pandemic Lockdown: A Case Study. 2020 IFEES World Engineering Education Forum - Global Engineering Deans Council (WEEF-GEDC), Engineering Education Forum - Global Engineering Deans Council (WEEF-GEDC), 2020 IFEES, 1-5. https://0-doi-org.biblioteca-ils.tec.mx/10.1109/WEEFGEDC49885.2020.9293681

Christenson S.L., Reschly A. L. \& Wylie C. (2012). Handbook of Research on Student Engagement (pp. 99). Springer.

Cohen, J., \& Jackson-Haub, D. (2019). Designing Learning for Student Engagement: An Online First Year Higher Education Experience. International Journal of Technologies in Learning, 26(2), 35-41. https://0-doi-org.biblioteca-ils.tec.mx/10.18848/2327-0144/CGP/v26i02/35-41

Conrad, R.-M., \& Donaldson, J. A. (2011). Engaging the online learner: activities and resources for creative instruction $\left(1^{\text {st }} \mathrm{ed}\right)$. Jossey-Bass. 
Fadde, P. J., \& Vu, P. (2014). Chapter 3: Blended Online Learning: Benefits, Challenges and Misconceptions. In J. C. Richardson, C. S. York, \& R. Patrick (Eds.), Online Learning: Common Misconceptions and Benefits and Challenges (pp. 33-47). Nova Science Publishers, Inc. https://0search-ebscohost-com.biblioteca-

ils.tec.mx/login.. spx ?direct=true $\& \mathrm{db}=\mathrm{e} 000 \mathrm{xww} \& \mathrm{AN}=714817 \&$ lang=es\&site=eds-live $\&$ scope $=$ site

Ferrer, J., Ringer, A., Saville, K., A Parris, M., \& Kashi, K. (2020). Students' motivation and engagement in higher education: the importance of attitude to online learning. Higher Education: The International Journal of Higher Education Research, 1. https://0-doi-org.bibliotecails.tec.mx/10.1007/s10734-020-00657-5

Henry, M. (2020). Online student expectations: A multifaceted, student-centred understanding of online education. Student Success, 11(2), 91-98. https://doi.org/10.5204/ssj.1678

Hernández Godoy, V.; Fernández Morales, K. y Pulido, J. (2018). La actitud hacia la educación en línea en estudiantes universitarios. Revista de Investigación Educativa, 36(2), 349-364. DOI: http://dx.doi.org/10.6018/rie.36.2.277451

Kanık, M. (2021). Students' Perception of and Engagement in Reactive Online Education Provided during the Covid-19 Pandemic. International Online Journal of Education \& Teaching, 8(2), $1063-$ 1082.

Lear, J.L., Ansorge, C., \& Steckelberg, A. (2010). Interactivity/Community Process Model for the Online Education Environment.

Lu, F., Chen, X., Ma, X., Liu, Z., \& Chen, Y. (2020). The Exploration and Practice of IT Solutions for Online Classes in Higher Education During COVID-19 Pandemic. 2020 International Symposium on Educational Technology (ISET), Educational Technology (ISET), 2020 International Symposium on, ISET, 298-302. https://0-doi-org.biblioteca-ils.tec.mx/10.1109/ISET49818.2020.00071

Martin, F., \& Bolliger, D. U. (2018). Engagement Matters: Student Perceptions on the Importance of Engagement Strategies in the Online Learning Environment. Online Learning, 22(1), 205-222.

Moore, M. J. (1993). Three types of interaction. In K. Harry, M. John, \& D. Keegan (Eds.), Distance education theory (pp. 19-24). New York: Routledge.

Montelongo, R. (2019). Less Than/More Than: Issues Associated with High Impact Online Teaching and Learning. Administrative Issues Journal Education Practice and Research. 9. 10.5929/9.1.5.

Kessels, U., Heyder, A., Latsch, M. \& Hannover, B. (2014) How gender differences in academic engagement relate to students' gender identity, Educational Research, 56:2, 220-229, DOI: 10.1080/00131881.2014.898916

Kovacs, H., Pulfrey, C., \& Monnier, E.-C. (2021). Surviving but not thriving: Comparing primary, vocational and higher education teachers' experiences during the COVID-19 lockdown. Education and Information Technologies: The Official Journal of the IFIP Technical Committee on Education, 1. https://0-doi-org.biblioteca-ils.tec.mx/10.1007/s10639-021-10616-x

Redacción CONECTA. (2020). Cancela Tec de Monterrey clases presenciales en prevención de COVID-19. CONECTA - Tecnologico de Monterrey. https://tec.mx/es/noticias/nacional/institucion/cancela-tec-de-monterrey-clases-presenciales-enprevencion-de-covid

Rhim, H.C., \& Han, H. (2020). Teaching online: foundational concepts of online learning and practical guidelines. Korean Journal of Medical Education, 32(3), 175-183.

Richardson, J. C., York, C. S., \& Lowenthal, P. R. (2014). Online Learning: Common Misconceptions and Benefits and Challenges. Nova Science Publishers, Inc.

Sahara, A., Mardji, Hadi, S., \& Elmaunsyah, H. (2021). Blended Learning Management in Higher Education Institutions in Indonesia. International Journal of Learning in Higher Education, 28(1), 65-73. https://0-doi-org.biblioteca-ils.tec.mx/10.18848/2327-7955/CGP/v28i01/65-73 
Tecnologico de Monterrey. (n.d.). Modelo Flexible y Digital. Transforma Tu Curso a Flexible y Digital Para La Continuidad Académica. https://innovacioneducativa.tec.mx/transforma-flexibledraft/modelo-flexible-digital/

Trochim, W. M. K., Donnelly, J. P., \& Arora, K. (2016). Research methods: the essential knowledge base (pp. 172, 222). Cengage Learning.

Volery, T. \& Lord, Deborah. (2000). Critical success factors in online education. International Journal of Educational Management. 14. 216-223. 10.1108/09513540010344731.

Wankel, C. \& Blessinger, P. (2013). Increasing Student Engagement and Retention in E-Learning Environments: Web 2.0 and Blended Learning Technologies: Vol. First edition. Emerald Group Publishing Limited.

Wood, D. R., \& Shirazi, D. S. (2020). A systematic review of audience response systems for teaching and learning in higher education: The student experience. Computers \& Education, 153. https://0-doiorg.biblioteca-ils.tec.mx/10.1016/j.compedu.2020.103896

You, Y. H., \& Beal, J. (2017). Mathematics: Online Teaching and Learning in Mathematics. In R. Alexander (Ed.), Título:Best Practices in Online Teaching and Learning Across Academic Disciplines. Fairfax, Virginia: George Mason University. 


\section{Appendix}

List of the survey questions applied to the Industrial and Systems Engineer students at Tecnologico de Monterrey Campus Sonora Norte:

Q1. I am motivated to be present in the class session.

Q2. I am motivated to participate and interact in the class session.

Q3. Under the Flexible Digital Model (MFD) my participation in class has been more active.

Q4. During your class, how often did you participate voluntarily?

Q5. In the Flexible Digital Model (MFD) I do not like to share my video in Zoom and I feel shamed

Q6. In your class, were there moments / activities that allowed you to interact with your teacher, ask him questions and have answers?

Q7. The frequency with which my teacher uses tools and technologies during my class is:

Q8. In your class, were there moments / activities where, through technology, you had the opportunity to comment, discuss, have a voice?

Q9. Activities through technological platforms (kahoot, stomboard, socrative, padlet, etc.) motivate you to participate more.

Q10. The activities through technological platforms where your name is not requested (menti, poll everywhere) motivate you to participate more.

Q11. In your class, were there moments / activities that ranged from individual work, to pair work, to teams with your colleagues?

Q12. In your class, were there moments where through technology you had the opportunity to contribute to activities with other classmates and be part of it?

Q13. I actively participate (solving exercises, discussing with colleagues, solving team activities, etc.).

Q14. How often we work in groups (using breakout rooms) on activities designed by my teacher.

Q15. How often did the sessions generate moments for the discussion of topics between you and your classmates?

Q16. Do you consider that the academic environment between you and your classmates encourages competitiveness?

Q17. The technological platform allowed you to feel truly involved and could participate and interact appropriately with your classmates and teacher in the synchronous sessions. 
Q18. The technological platform used for the synchronous sessions of your class made you feel comfortable / safe to express your opinions and / or doubts.

Q19. The frequency with which my teacher explains course content and / or solves exercises.

Q20. How often my teacher asks us to read or view material before class sessions and asks us to complete a type of test or quiz before class starts.

Q21. How often did you get feedback from your teacher?

Q22. In your class, were there moments / activities that made you realize the degree of progress in your learning and hooked you to continue in that process?

Q23. In your class, were there moments / activities that led you to self-assess and feedback your own learning, and reflect on it?

Q24. How often did the teacher ask to do a reflection activity at the end of class; for example, asking questions, expressing our learning?

Q25. I adjusted in my study method by consulting the class videos, study materials and feedback provided by the teacher to better understand the topics.

Q26. I discovered the need to seek and consult additional information to the material provided by the teacher.

Q27. Do you think that the teacher correctly adapted the pace and content of the class with respect to the performance that the group was showing? 